\title{
RESEARCH
}

Open Access

\section{Circular RNA FOXP1 promotes tumor progression and Warburg effect in gallbladder cancer by regulating PKLR expression}

Shouhua Wang ${ }^{1}$, Yongjie Zhang ${ }^{2}$, Qiang Cai ${ }^{1}$, Mingzhe Ma ${ }^{3}$, Long Yang Jin ${ }^{1}$, Mingzhe Weng ${ }^{1}$, Di Zhou ${ }^{1}$, Zhaohui Tang $^{1 *}$, Jian Dong Wang ${ }^{1 *}$ and Zhiwei Quan ${ }^{1^{*}}$ (D)

\begin{abstract}
Background: Circular RNAs (circRNAs) have recently been identified as potential functional modulators of the cellular physiology processes. The study aims to uncover the potential clinical value and driving molecular mechanisms of circRNAs in gallbladder cancer (GBC).

Patients and methods: We performed RNA sequencing from four GBC and paired adjacent normal tissues to analyze the circRNA candidates. Quantitative real-time polymerase chain reaction (QRT-PCR) was used to measure the circFOXP1 expression from 40 patient tissue samples. Short hairpin RNA mediated knockdown or exogenous expression of circFOXP1 combined with in vitro and in vivo assays were performed to prove the functional significance of circFOXP1. Double luciferase reporter, RNA immunoprecipitation (RIP) and RNA pull-down assays were also performed.

Results: By performing RNA sequencing from GBC and paired adjacent normal tissues to analyze the circRNA candidates, we identified that circFOXP1 (hsa_circ_0008234) expression was significantly upregulated in GBC tissues and positively associated with lymph node metastasis, advanced TNM stage and poor prognosis in patients. Short hairpin RNA mediated knockdown or exogenous expression of circFOXP1 combined with in vitro assays demonstrated that circFOXP1 has pleiotropic effects, including promotion of cell proliferation, migration, invasion, and inhibition of cell apoptosis in GBC. In vivo, circFOXP1 promoted tumor growth. Mechanistically, double luciferase reporter, RNA immunoprecipitation (RIP) and biotin-labeled RNA pull-down assays clarified that circFOXP1 interacted with PTBP1 that could bind to the $3^{\prime} \mathrm{UTR}$ region and coding region (CDS) of enzyme pyruvate kinase, liver and RBC (PKLR) mRNA (UCUU binding bites) to protect PKLR mRNA from decay. Additionally, circFOXP1 acted as the sponge of miR-370 to regulate PKLR, resulting in promoting Warburg effect in GBC progression.
\end{abstract}

Conclusions: These results demonstrated that circFOXP1 serve as a prognostic biomarker and critical regulator in GBC progression and Warburg effect, suggesting a potential target for GBC treatment.

Keywords: Gallbladder cancer, circFOXP1, PTBP1, PKLR, miR-370

\footnotetext{
* Correspondence: tangzhaohui@xinhuamed.com.cn;

wangjiandong@xinhuamed.com.cn; quanzhiwei@xinhuamed.com.cn

'Department of General Surgery, Xinhua Hospital, Shanghai Jiao Tong

University School of Medicine, No.1665 Kong jiang Road, Yangpu District,

Shanghai 200000, China

Full list of author information is available at the end of the article
}

(c) The Author(s). 2019 Open Access This article is distributed under the terms of the Creative Commons Attribution 4.0 International License (http://creativecommons.org/licenses/by/4.0/), which permits unrestricted use, distribution, and reproduction in any medium, provided you give appropriate credit to the original author(s) and the source, provide a link to the Creative Commons license, and indicate if changes were made. The Creative Commons Public Domain Dedication waiver (http://creativecommons.org/publicdomain/zero/1.0/) applies to the data made available in this article, unless otherwise stated. 


\section{Background}

Gallbladder cancer (GBC) is the most common and leading cause of cancer-associated mortality among biliary tract carcinomas worldwide. Complete surgical resection is the only potentially curative choice for $\mathrm{GBC}$, but due to lack of specific signs, symptoms or reliably sensitive disease markers, many patients are diagnosed at advanced stages [1, 2]. Conventional chemotherapy, radiotherapy, and molecular targeted therapy provide palliative relief for GBC patients; however, in the past few decades, there has been no definitive improvement in overall survival (OS) [3-5]. Therefore, improving molecular understanding of the underlying pathogenesis in GBC and investigating mechanism-based therapeutic strategies for patients is essential.

Circular RNAs (circRNAs), covalently closed RNAs formed from both exonic and intronic sequences, are highly stable RNA molecules [6, 7]. CircRNAs exert certain functions, including as intermediates in RNA alternative splicing (AS), as regulators of transcription in cis, and as miRNA sponges [8]. Some of circRNAs are identified as potential functional modulators of cellular physiology processes involved in cell proliferation, differentiation, apoptosis and tumor progression [9, 10]. For example, CiRS-7 (also termed CDR1as) harbors more than 70 conventional miR-7 binding sites and is involved in cancer-related pathways [11]. CircHIPK3 but not HIPK3 mRNA regulates human cell growth in hepatocellular carcinoma [12]. Ectopic expression of circ-foxo3 repressed cell cycle progression by binding to cyclindependent kinase 2 (CDK2) and cyclin-dependent kinase inhibitor 1 (or p21) [13].

The Warburg effect (or aerobic glycolysis) is a wellcharacterized metabolic alteration that involved in cancer phenomenon including rapid cell proliferation, invasion, and migration in tumors [14]. enzyme pyruvate kinase, liver and RBC (PKLR) is identified as a pivotal regulator of glycolytic reprogramming and affect tumor cells. Nie et al. showed that mineralocorticoid receptor (MR) inhibits the Warburg effect and cancer progression via the miR-3383p-PKLR axis in hepatocellular carcinoma [15]. Yang et al. demonstrated the NQO1/PKLR axis promotes lymph node metastasis and breast cancer progression by activating the AMPK and AKT/mTOR signaling pathway and consequently induced glycolytic reprogramming [16]. The association of between circRNAs and Warburg effect in gallbladder cancer remains unknown.

Here, we identified a circular FOXP1 RNA (circFOXP1, hsa_circ_0008234), derived from the exon region of the FOXP1 gene $[17,18]$, which is significantly upregulated in GBC tissues. CircFOXP1 interacted with PTBP1 or sponged miR-370, which promoted tumor progression and the Warburg effect in GBC by directly targeting the PKLR. Our results indicate that
circFOXP1 may be a novel potential target for GBC treatment.

\section{Methods \\ RNA-sequencing analysis}

A detailed description of RNA-sequencing analysis was provided in Additional file 1: Supplementary Materials and Methods section. The GEO Accession number is GSE100363.

\section{Patient tissue samples}

Forty human GBC and adjacent normal tissue specimens were collected from patients who underwent radical resection at Xinhua Hospital between March 2009 and January 2013. The eleven patients were male and twenty-nine were female. Ages ranged from 35 to 82 years (the mean value $=$ 54.85 years). Each tissue sample was snap-frozen in liquid nitrogen for further analysis. All of patients in this study belonged to the same ethnic group. The patients were selected according to the criteria: (1) All clinicopathological diagnoses were confirmed by two pathologists. (2) None of the patients received any treatments before surgery. (3) None of the patients received radiotherapy or chemotherapy during follow-up period. (4) availability of complete follow-up data and not lost follow-up. (5) no death in the perioperative period. (6) no history of other synchronous malignancies. (7) For patients undergoing radical resection of gallbladder cancer. The study procedure was approved by the Human Ethics Committee of Xinhua Hospital. All patients signed consent forms. Follow-ups after surgery were performed according to patient survival time until March 8, 2016. The clinicopathological data were shown in Table 1.

\section{Cell culture}

Human GBC cell lines NOZ, GBC-SD, EHGB-1, SGC-996 and OCUG-1 and the human intrahepatic biliary epithelial cell line H69 and another normal biliary epithelia cell line HIBEC were used in the present study. GBC-SD and OCUG-1 cell lines were purchased from Cell Bank of the Chinese Academy of Science (Shanghai, China). The NOZ cell line was purchased from the Health Science Research Resources Bank (Osaka, Japan). EHGB-1 and SGC-996 cells were a generous gift from Eastern Hepatobiliary Surgical Hospital and Institute, The Second Military University, Shanghai, China. The cells were cultured in Dulbecco's modified Eagle's medium (Gibco BRL, Grand Island, NY, USA); the cell media contained $10 \%$ fetal bovine serum (FBS, HyClone, Invitrogen). Cells were maintained in a humidified incubator at $37{ }^{\circ} \mathrm{C}$ in the presence of $5 \% \mathrm{CO}_{2}$.

\section{RNAi, plasmid construction and cell transfection}

Cells were transfected using lipofectamine 2000 according to the manufacturer's instructions. Additional information 
Table 1 Correlation between circFOXP1 expression and clinicopathological characteristics in 40 cases GBC patients

\begin{tabular}{|c|c|c|c|c|}
\hline \multirow[b]{2}{*}{ Clinicopathological characteristics } & \multirow[b]{2}{*}{ The number of patients } & \multicolumn{2}{|c|}{ CircFOXP1 expression } & \multirow[t]{2}{*}{$P$-value } \\
\hline & & $\begin{array}{l}\text { Lower } \\
(n=20)\end{array}$ & $\begin{array}{l}\text { Higher } \\
(n=20)\end{array}$ & \\
\hline \multicolumn{5}{|c|}{ Age } \\
\hline$\leq 60$ & 25 & 10 & 15 & \multirow[t]{2}{*}{0.102} \\
\hline$>60$ & 15 & 10 & 5 & \\
\hline \multicolumn{5}{|c|}{ Gender } \\
\hline Male & 11 & 6 & 5 & \multirow[t]{2}{*}{0.723} \\
\hline Female & 29 & 14 & 15 & \\
\hline \multicolumn{5}{|c|}{ Tumor size } \\
\hline$<5 \mathrm{~cm}$ & 16 & 11 & 5 & \multirow[t]{2}{*}{0.053} \\
\hline$\geq 5 \mathrm{~cm}$ & 24 & 9 & 15 & \\
\hline \multicolumn{5}{|l|}{ Histological grade } \\
\hline well and moderately & 21 & 10 & 11 & \multirow[t]{2}{*}{0.752} \\
\hline Poorly and others & 19 & 10 & 9 & \\
\hline \multicolumn{5}{|l|}{ Lymph node metastasis } \\
\hline NO & 18 & 13 & 5 & \multirow[t]{2}{*}{$0.011^{*}$} \\
\hline $\mathrm{N} 1 / 2$ & 22 & 7 & 15 & \\
\hline TNM stage & & & & $0.027^{*}$ \\
\hline$|-| \mid$ & 19 & 13 & 6 & \\
\hline III-IV & 21 & 7 & 14 & \\
\hline Adjacent organ invasion & & & & 0.110 \\
\hline No & 17 & 11 & 6 & \\
\hline Yes & 23 & 9 & 14 & \\
\hline
\end{tabular}

${ }^{*} P<0.05 . T N M$ tumor-node-metastasis

about the performed experiments can be found in the Additional file 1: Supplementary Materials and Methods. The sequences of all oligonucleotide used in the study were shown in Additional file 2: Table S1.

\section{Cell proliferation, migration and invasion assays and cell cycle and cell apoptosis analysis}

Cell proliferation was assessed using Cell Counting Kit 8 (Dojindo, Japan); cell migration and invasion was assessed with transwell assays. Cell cycle distribution and cell apoptosis rate analysis was analyzed using flow cytometry. Detailed descriptions of experiments can be found in Additional file 1: Supplementary Materials and Methods.

\section{RNA isolation, quantitative real-time PCR (qRT-PCR) and western blot analysis}

RNA isolation, qRT-PCR and western blot analysis were performed as described previously [19]. Detailed descriptions of experiments can be found in Additional file 1:
Supplementary Materials and Methods. The sequences of all primers used in the study were shown in Additional file 2: Table S1.

\section{Xenograft mouse model}

NOZ cells $\left(1 \times 10^{6}\right)$ stably expressing sh-NC, shcircFOXP1-1, sh-circFOXP1-2 or GBC-SD cells $(1 \times$ $10^{6}$ ) stably expressing pLCDH-vector or pLCDHcircFOXP1 were subcutaneously injected into either side of the flank area of 3-week-old male nude mice $(n=5$ mice per group). Tumor volumes were measured $\left(0.5 \times\right.$ length $\times$ width $\left.^{2}\right)$ and tumor weights were evaluated in mice on a weekly basis. After 4 weeks, mice were sacrificed, and the tumors were excised. The study protocol was approved by the Animal Care and Use committee of Xinhua Hospital (approval ID: 2014041). All animal experiments were performed in the animal laboratory center at Xinhua Hospital and conformed to the Guide for the Care and Use of Laboratory Animals published by the US National 
Institutes of Health (NIH publication number 85-23, revised 1996).

\section{RNA pull-down assay}

RNA pull-down assays were performed as described previously [20, 21]. Briefly, cells extract $(2 \mu \mathrm{g})$ was mixed with biotinylated RNA (100 pmol). Washed streptavidin agarose beads $(100 \mathrm{ml})$ were added to each binding reaction and further incubated at room temperature for $1 \mathrm{~h}$. Beads were washed briefly three times and boiled in SDS buffer, and the retrieved protein was determined by western blot analysis.

\section{RNA immunoprecipitation (RIP) assay}

RIP assays were performed using an EZ-Magna RIP ${ }^{\mathrm{mi}}$ RNA-Binding Protein Immunoprecipitation Kit (Millipore, Billerica, MA, USA) according to the manufacturer's instructions. Cells at approximately $90 \%$ confluence was lysed using complete RIP lysis buffer containing RNase Inhibitor (Millipore) and protease inhibitor and then $100 \mu \mathrm{l}$ of whole cell extract was incubated with RIP buffer containing magnetic beads conjugated to specific antibodies. The negative control was normal mouse anti-IgG antibody (Cell Signaling Technology, USA), and the positive control was anti-SNRNP70 antibody (Millipore, USA).

\section{Statistical analysis}

All experiments were independently repeated at least three times. Statistical analyses were performed using SPSS 20.0 (SPSS, Chicago, IL, USA). The data are expressed as the mean \pm standard error of the mean (SEM). OS was estimated using the Kaplan-Meier method and a log-rank test. The difference between groups was analyzed using Student's t-test. Differences were statistically significant at $P<0.05$.

\section{Results}

\section{Expression of circFOXP1 is significantly upregulated in} GBC tissues and cells

To explore circRNAs expression profile in GBC, we performed RNA sequencing analyses of ribosomal RNAdepleted total RNA from four pairs of GBC tissues and adjacent normal tissues. The cluster heat map demonstrated the differentially expressed circRNAs over 3.5fold change (Fig. 1a, left). The scatter plots showed that upregulated 44 circRNAs and downregulated 81 circRNAs in GBC tissues compared with adjacent normal tissues (Fig. 1a, right). Compared with previously reported database obtained from circBase [18], 34 of the top 44 upregulated circRNAs were overlapped circRNAs and were listed in Fig. 1a, left. Several circRNAs including circXPO1 (chr2_61710091_61717911_-, hsa_circ_ 0002607), circFOXP1 (chr3_71090478_71102924_-,hsa circ_0008234),circMAPK1(chr22_22153300_22162135_-, hsa_circ_0004872), circSENP1 (chr12_48477373_ 48491907_-, hsa_circ_0006222) and circSMAD2 (chr18_ 45391429_45423180_-, hsa_circ_0000847) were chosen for further investigation because their parental genes are involved in cell proliferation, invasion and cell cycle regulation in some tumors [22-26]. We examined these investigated circRNA candidates in GBC tissues and paired normal tissue samples from 40 patients. The results showed that expression of circFOXP1, circMAPK1 and circSAMD2 were upregulated in GBC tissues (Fig. $1 \mathrm{~b}$, top and Additional file 3: Figure S1A). GBC patients were classified into two groups: high-circFOXP1 group $(n=20$, circFOXP1 expression ratio $\geq$ median ratio $)$ and low-circFOXP1 group ( $n=20$, circFOXP1 expression ratio $<$ median ratio). Kaplan-Meier analysis and log rank tests indicated that higher circFOXP1 expression levels were positively correlated with a shorter OS in patients (Fig. 1b, low), while circSMAD2 and circMAPK1 expression showed no statistical significance with $O S$ in patients (Additional file 3: Figure S1B). CircFOXP1 expression was upregulated in several tumors compared with normal tissues through RNA sequencing data in a previous study [12]. However, the clinical significance and functional role of circFOXP1 remains unknown.

As seen in Table 1, the statistical analysis revealed that high circFOXP1 expression levels were strongly correlated with lymph node metastasis $(P=0.011)$ and advanced TNM stage (III-IV) $(P=0.027)$ in GBC patients, but no correlation was found with the other factors (Table 1). Univariate and multivariate Cox analysis revealed that lymph node metastasis $(\mathrm{HR}=3.749,95 \% \mathrm{CI}$ : $1.578-8.905, P<0.05)$ and high circFOXP1 expression $(\mathrm{HR}=3.658,95 \% \mathrm{CI}: 1.587-8.431, \mathrm{P}<0.05)$ were independent risk factors for the OS of GBC patients (Table 2). As shown in Additional file 4: Figure S2A, the transcript levels of circFOXP1 were also significantly higher in several GBC cells than in the bile duct epithelial cell line H69 or another normal biliary epithelia cell line HIBEC. RNA was isolated and RT-PCR with Sanger sequencing confirmed the circular form in GBC cells (Additional file 4: Figure S2B). RNA isolated from the above cell lines was subjected to northern blot using a probe specific for circFOXP1, confirming that circFOXP1 was present in these cells (Fig. 1c). In addition, we confirmed that circFOXP1 was resistant to RNase $\mathrm{R}$ after digestion of RNA using RNase $\mathrm{R}$ exonuclease (Fig. 1d and Additional file 4: Figure S2C), which was consistent with a previous study [27], the circHIPK3 was used as the control, which was reported as a circRNA in previous study [12]. Using fluorescence in situ hybridization (FISH) and qRT-PCR analysis, we also noted that circFOXP1 localized in the cytoplasm and nucleus, but was predominately enriched in the cytoplasm in GBC cells (Fig. 1e and Additional file 4: Figure S2D). Thus, the 


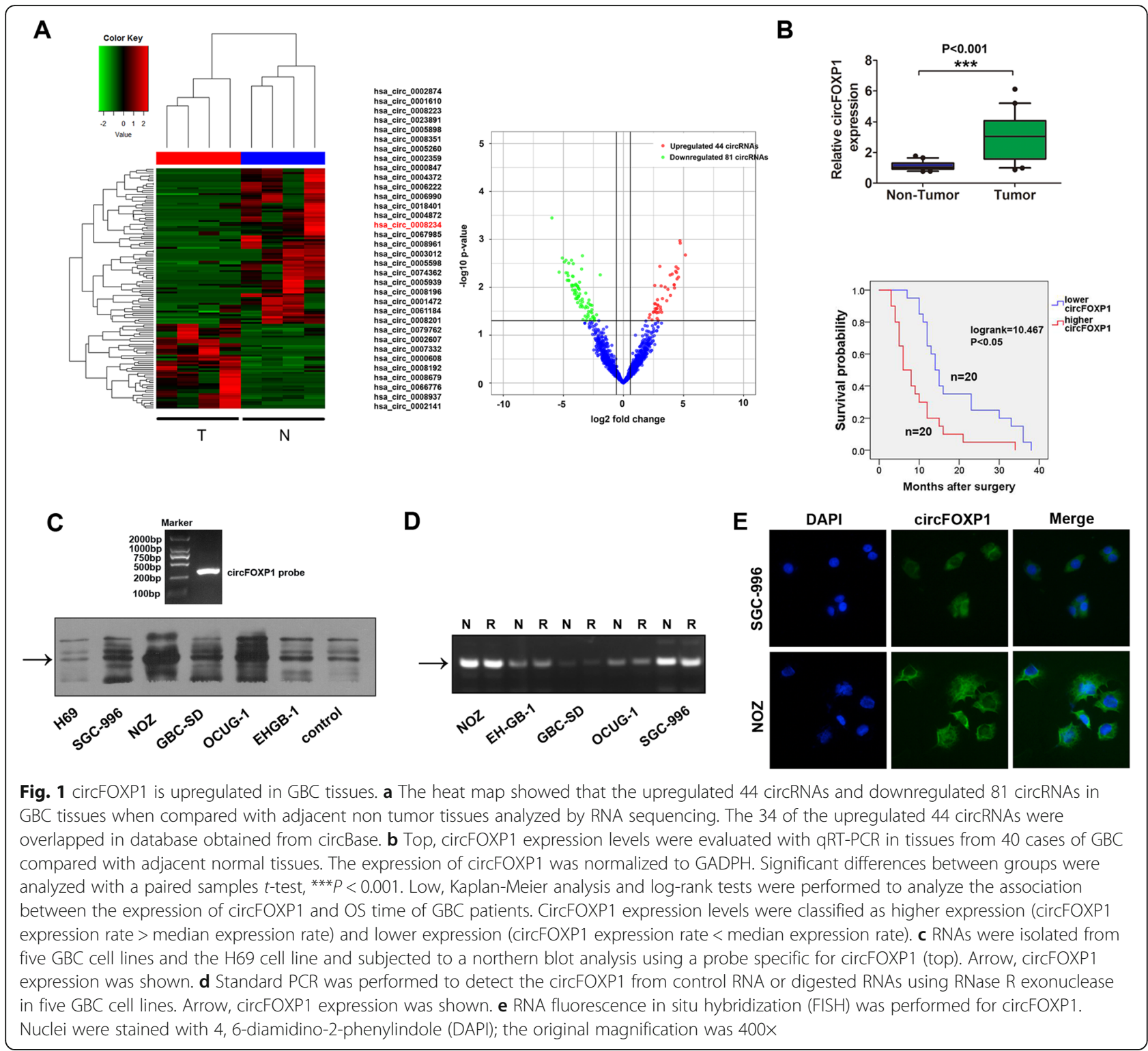

Table 2 Univariate and multivariate Cox analysis of the overall survival (OS) in 40 GBC patients

\begin{tabular}{|c|c|c|c|c|c|c|}
\hline \multirow[t]{2}{*}{ Factors } & \multicolumn{3}{|c|}{ Univariate Cox analysis } & \multicolumn{3}{|c|}{ Multivariate Cox analysis } \\
\hline & $\overline{\mathrm{HR}}$ & $95 \% \mathrm{Cl}$ & $P$-value & $\overline{\mathrm{HR}}$ & $95 \% \mathrm{Cl}$ & $P$-value \\
\hline Age & 1.005 & $0.974-1.037$ & 0.754 & & & \\
\hline Gender & 0.796 & $0.390-1.624$ & 0.530 & & & \\
\hline Tumor size & 0.917 & $0.484-1.738$ & 0.790 & & & \\
\hline Histological grade & 1.426 & $0.755-2.693$ & 0.274 & & & \\
\hline Lymph node metastasis & 2.938 & $1.476-5.848$ & $0.002^{*}$ & 3.749 & $1.578-8.905$ & $0.003^{*}$ \\
\hline TNM stage & 2.105 & $1.064-4.164$ & $0.033^{*}$ & 1.123 & $0.372-2.032$ & 0.747 \\
\hline Adjacent organ invasion & 1.985 & $1.003-3.930$ & $0.049^{*}$ & 0.968 & $0.447-2.317$ & 0.896 \\
\hline Higher circFOXP1 & 2.762 & $1.422-5.362$ & $0.003^{*}$ & 3.658 & $1.587-8.431$ & $0.002^{*}$ \\
\hline
\end{tabular}


above results indicated that circFOXP1 may serve as a prognostic biomarker for GBC.

\section{Overexpression of circFOXP1 promotes GBC cell growth in vitro and in vivo}

To further explore the biological significance of circFOXP1 in GBC progression, gain-and loss-of-function studies were performed. Based on endogenous expression of circFOXP1 in several GBC cell lines, we developed NOZ and SGC-996 cells with circFOXP1 stably silenced by shRNA-circFOXP1 and EHGB1 or GBCSD cells with circFOXP1 stably overexpressed using pLCDH-circFOXP1. Meanwhile, to confirm the specificity of circFOXP1 silencing or overexpression, we showed that the cells transfection had no effects on mRNA expression of FOXP1 in GBC cells. In addition, circFOXP1 was also overexpressed after digestion of RNA using RNase $\mathrm{R}$ by using pLCDH-circFOXP1 (Additional file 4: Figure S2E-2F). By performing CCK8 assays and flow cytometry analysis in vitro, we observed that knockdown of endogenous circFOXP1 significantly suppressed cell proliferation capacity, G1$\mathrm{S}$ arrest, and increased the cell apoptosis rate in $\mathrm{NOZ}$ and SGC-996 cells, but upregulated expression of circFOXP1 dramatically promoted the cell proliferation capacity, beyond the G1-S transition, and reduced the cell apoptosis rate in GBC-SD cells (Fig. 2a-c). Consistent with the decreased cell proliferation capacity, $\mathrm{NOZ}$ and SGC-996 cells exhibited reduced expression levels of proliferating cell nuclear antigen (PCNA), MMP9 and AKT, but increased Caspase- 3 expression after knockdown of endogenous circFOXP1, and conversely, upregulated expression of circFOXP1 had the opposite effects in GBC cells (Additional file 5: Figure S3A). As shown in Additional file 5: Figure S3B-3C, migration and invasion capacities were impaired in $\mathrm{NOZ}$ and SGC-996 cells after knockdown of endogenous circFOXP1, but were dramatically promoted in GBC-SD cells after circFOXP1 overexpression.

To evaluate the biological function of circFOXP1 in vivo, a xenograft tumor model was constructed by inoculating different clones of NOZ and GBC-SD cells subcutaneously into nude mice. The results confirmed that the mean tumor volumes and weights were larger and tumor growth was rapid in the upregulated expression of circFOXP1 group, compared with the control group. However, the tumor growth was inhibited, as shown by decreasing mean volumes and weights, in the knockdown of endogenous circFOXP1 group compared with the control group (Fig. 2d). Immunohistochemical staining of tumor tissues revealed an increased proportion of proliferating cells (Ki67+) in the upregulated expression of circFOXP1 group compared with the control group. In contrast, knockdown of endogenous circFOXP1 led to reduced expression of Ki67 compared with the control group (Fig. 2e). These results indicated that circFOXP1 promoted GBC growth in vitro and in vivo.

\section{Upregulation of circFOXP1 promotes the Warburg effect in GBC cells}

The Warburg effect, characterized by abnormal metabolic phenomena that enhance glycolysis and reduce oxidative phosphorylation, induces significant differences between cancer cells and normal cells and affects tumor progression [28]. Based on the above findings, we investigated whether the expression of circFOXP1 affected the Warburg effect by measuring the levels of pyruvate production and lactate production and the glycolytic rate in GBC cells. The statistical analysis revealed a dramatically lower level of lactate production and pyruvate production after circFOXP1 knockdown in NOZ and SGC-996 cells, conversely, were significantly higher after circFOXP1 overexpression in GBC-SD cells (Fig. 3a). Furthermore, glycolysis and extracellular acidification rates (ECAR) were analyzed using an XF24 Extracellular Flux analyzer (Seahorse), The results confirmed that circFOXP1 knockdown reduced the ECAR in NOZ and SGC-996 cells, while upregulated circFOXP1 expression increased ECAR in GBCSD cells (Fig. 3b-c). we also measured oxygen consumption rate (OCR, a marker of OXPHOS) and found that knockdown of circFOXP1 enhanced OCR ability in NOZ and SGC-996 cells, in contrast, upregulated circFOXP1 expression reduced OCR ability in GBC-SD cells (Fig. 3d-e). The results confirmed that circFOXP1 knockdown impaired the glycolysis rate and glycolytic capacity in NOZ and SGC-996 cells. On the other hand, upregulated circFOXP1 expression significantly enhanced the rate of glycolysis and glycolytic capacity in GBC-SD cells (Fig. 3f-g). In addition, knockdown of circFOXP1 in NOZ and SGC-996 cells also elevated the formation of ATP produced by OXPHOS, but upregulated circFOXP1 has an decreased formation of ATP in GBC-SD cells (Fig. 3g). These results indicated that upregulation of circFOXP1 promoted the Warburg effect in GBC cells.

\section{The RNA-binding protein PTBP1 binds to circFOXP1 in $\mathrm{GBC}$ cells}

We further investigated the mechanisms by which circFOXP1 promoted the Warburg effect in GBC cells. Recent evidence has indicated that circRNAs participate in molecular regulation by interacting with several proteins [13]. Based on this hypothesis, we identified circFOXP1interacting proteins by performing an RNA pull-down assay combined with Liquid Chromatography-Mass Spectrometry (LC-MS) in NOZ cells. The RNA-related proteins were determined using SDS-polyacrylamide gel electrophoresis (SDS/PAGE) and silver staining (Fig. 4a). By using LC-MS and comparing the results with those from antisense circFOXP1 experiments, proteins were 


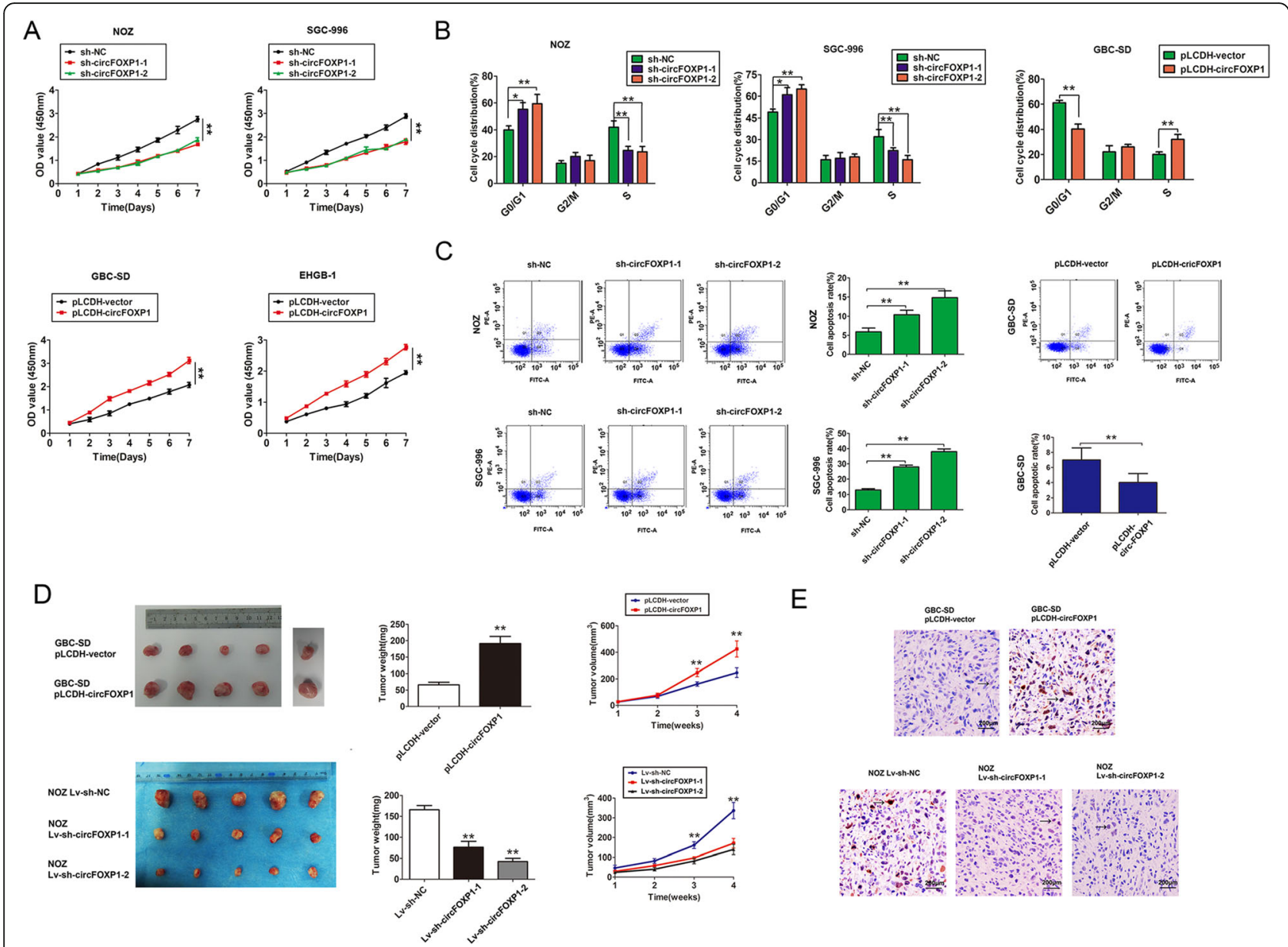

Fig. 2 circFOXP1 promotes cell proliferation in vitro and in vivo. a Cell proliferation capacity was evaluated with CCK8 assays. Briefly, 2000 cells/ well were plated in triplicate and cell proliferation was detected at days 1, 2, 3, 4, 5, 6 and 7 days after transfection of NOZ and SGC-996 cells (top) with sh-NC, sh-circFOXP1-1 or sh-circFOXP1-2 or of GBC-SD and EHGB-1 cells (low) with pLCDH-vector and pLCDH-circFOXP1. The experiment was repeated three times, ${ }^{*} P<0.01$. $\mathbf{b}$ Data are presented as the percentage cell phase distribution including G0/G1, S and G2/M phases after transfection of NOZ and SGC-996 cells with sh-NC, sh-circFOXP1-1 or sh-circFOXP1-2 or GBC-SD cells with pLCDH-vector and pLCDH-circFOXP1, ${ }^{*} P<0.05 ;{ }^{*} P<0.01$. c The data are presented as cell apoptosis rates after transfection of NOZ and SGC-996 cells (left) with shNC, sh-circFOXP1-1 or sh-circFOXP1-2 or GBC-SD cells (right) with pLCDH-vector and pLCDH-circFOXP1. ${ }^{* * P}<0.01$. $\mathbf{d}$ Tumor weight and volume were detected to monitor tumor growth in subcutaneous implantation mouse models; mice were implanted with GBC-SD cells (top) transfected with pLCDH-vector or pLCDH-circFOXP1 and NOZ cells (low) transfected with Iv-sh-NC, Iv-sh-circFOXP1-1 or Iv-sh-circFOXP1-2. e Immunohistochemical staining of Ki67 expression was shown in tumor tissues, as indicated by the number of Ki67-positive cells with GBC-SD cells (top) transfected with pLCDH-vector or pLCDH-circFOXP1 and NOZ cells (low) transfected with Iv-sh-NC, Iv-sh-circFOXP1-1 or Iv-shcircFOXP1-2, (original magnification, 200x)

identified by LC-MS that specifically associated with circFOXP1 (Additional file 6: Table S2). PTBP1 was further detected due to higher expression in the circFOXP1sense probe compared with the circFOXP1-antisense sample or no RNA sample, and previous studies have verified that PTBP1 is associated with the Warburg effect in cancer cells through regulation of the PKM1/ PKM2 ratio [29]. Western blot analysis with anti-PTBP1 antibody indicated the existence of PTBP1 within the circFOXP1 sense RNA probe pull-down samples in NOZ and SGC-996 cells (Fig. 4b). Meanwhile, a RIP assay with PTBP1 antibody showed that endogenous
PTBP1 directly bound to circFOXP1 in NOZ and SGC996 cells (Fig. 4c). Moreover, we observed that knockdown of endogenous circFOXP1 decreased the protein expression of PTBP1 in NOZ and SGC-996 cells, but upregulated expression of circFOXP1 upregulated the expression of PTBP1 in GBC-SD cells (Fig. 4d). By Immunofluorescence and western blot analysis, we further demonstrated that exogenous expression of circFOXP1 enhanced expression of PTBP1 by increased transportation levels of PTBP1 from the nucleus to the cytoplasm, but knockdown of circFOXP1 also revert PTBP1 back to the nucleus (Fig. 5a-b). 


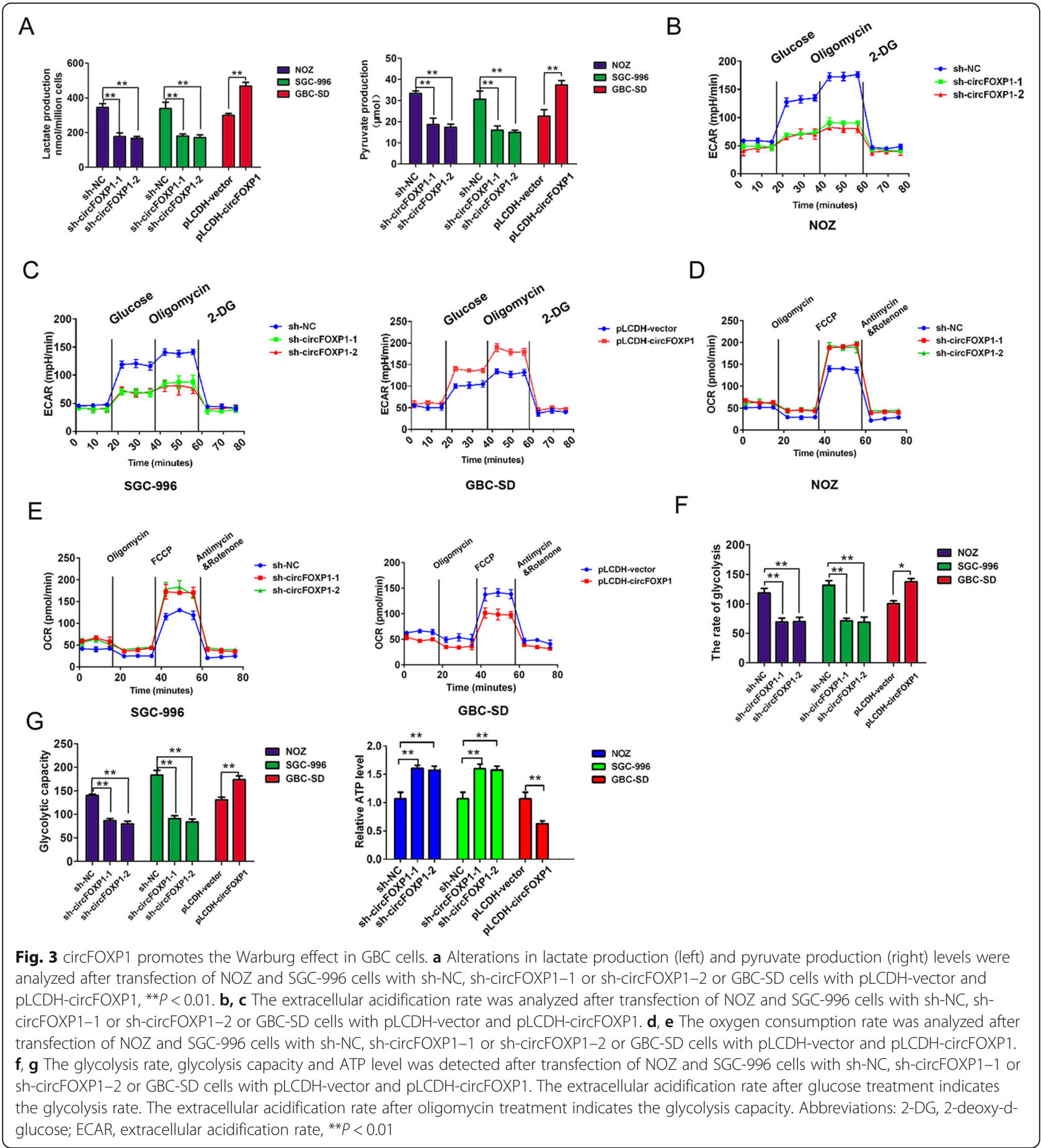

\section{PTBP1 promotes PKLR mRNA expression in GBC cells}

In mammalian cells, pyruvate kinase is encoded by 4 isozymes: M1, M2, liver (PKL), and red blood cell (PKR). While the M1, PKL, and PKR isozymes are described to exhibit tissue-specific expression, the pyruvate kinase M2 isoform is highly expressed across cancer types. Glycolytic deregulation, including activation of upregulated PKM2 and liver and RBC (PKLR), is a driver that promotes cancer progression $[15,30,31]$. Previous reports confirmed that PTBP1 regulates AS of the pyruvate kinase gene (PKM) in the acquisition of oncogenic features by increasing recruitment to the PKM premRNA to promote PKM2 splicing, which contributes to the Warburg effect [32]. First, we speculated whether decreased circFOXP1 led to exertion of the function of PTBP1 to regulate AS. The results confirmed that 

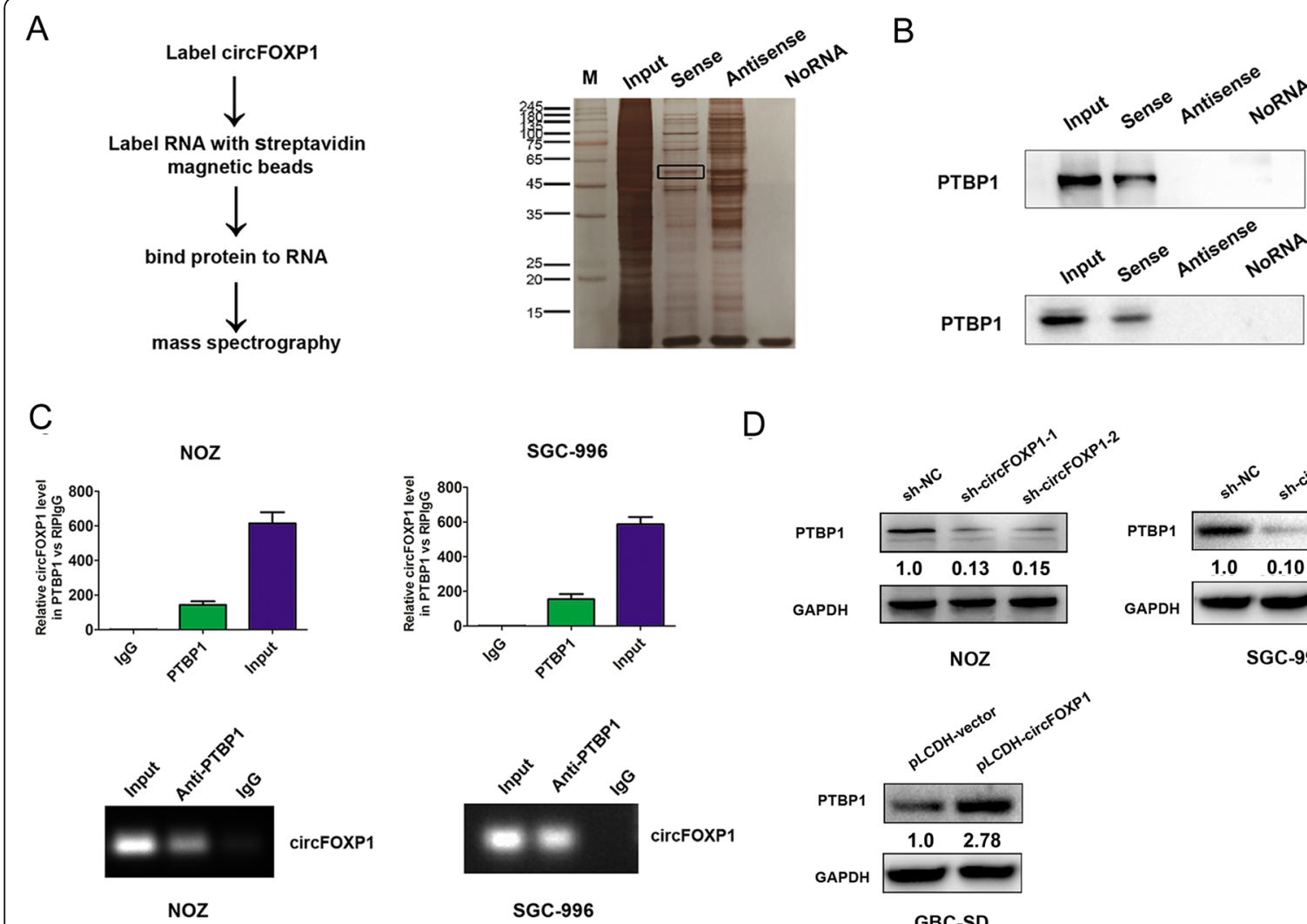

NOZ

SGC-996

C
SGC-996

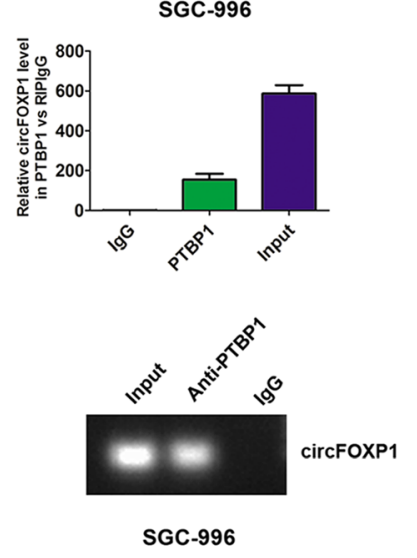

D
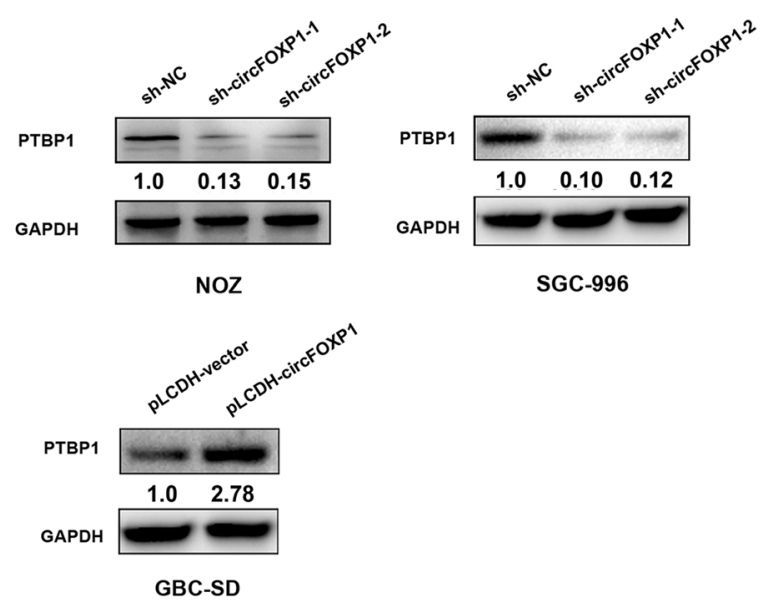

Fig. 4 circFOXP1 interacts with PTBP1 in GBC cells. a A RNA pull-down assay was performed (left) and the RNA-related proteins were determined with SDS-polyacrylamide gel electrophoresis (SDS/PAGE) and silver staining (right). b PTBP1 was pulled down by a circFOXP1 sense RNA probe but not by an antisense RNA probe or no RNA in NOZ and SGC-996 cells. Western blot analysis was performed to detect the specific association of PTBP1 and circFOXP1 $(n=3)$ ). c RIP assays with qRT-PCR (top) or RT-PCR (low) showed that circFOXP1 was pulled down by an anti-PTBP1 antibody in NOZ and SGC-996 cells $(n=3)$. $\mathbf{d}$ The relative protein expression of PTBP1 after transfected with sh-NC, sh-circFOXP1-1 or shcircFOXP1-2 in NOZ and SGC-996 cells and transfected with pLCDH-vector or pLCDH-circFOXP1 in GBC-SD cells were determined by western blot analysis $(n=3)$. All data are shown as mean \pm S.E.M., $\mathrm{n}=3$ or more, ${ }^{* *} P<0.01$

knockdown of endogenous circFOXP1 resulted in no significant change in the mRNA and protein levels of PKM1 in NOZ and SGC-996 cells but had a partial effect on the PKM2 protein level (Fig. 5c-d). PKLR is also known as a promoter in modulation of the Warburg effect [15]. Interestingly, dramatically decreased PKLR expression levels were observed after downregulation of circFOXP1 in NOZ and SGC-996 cells or in NOZ cells in vivo, but increased expression of PKLR was observed after upregulation of circFOXP1 in GBC-SD cells or in vivo (Fig. 5e-f and Additional file 7: Figure S4A). In addition, we showed that PKLR silencing impaired the glycolysis rate and glycolytic capacity in NOZ and SGC996 cells, which inhibited the Warburg effect in GBC (Additional file 7: Figure S4B-4F).

PKLR acts as a driver of tumor growth and metastasis $[15,33]$. We sought to elucidate the underlying mechanism by which circFOXP1 affect PKLR expression. PTBP1, an RNA-binding protein, exerts various molecular functions, including RNA metabolism, for example, control of mRNA stability or degradation [34, 35], determination of mRNA localization [36], and protection of mRNAs from decay [37]. Compared with normal tissues, PTBP1 was upregulated in gallbladder cancer tissues and the protein and mRNA of PKLR was also upregulated in gallbladder cancer tissues (Fig. 6a). Furthermore, we showed that the proteasome inhibitor MG-132 could increase PKLR expression and abolished the reduction in PKLR protein levels in circFOXP1-knockdown NOZ cells (Additional file 8: Figure S5A). The protein synthesis inhibitor cycloheximide ( $\mathrm{CHX}$ ) decreased the expression of PKLR proteins by inhibiting protein synthesis, but upregulation of PKLR in circFOXP1-overexpressing SGC-996 and GBC-SD cells was not abolished by treatment with CHX (Additional file 8: Figure S5B). These results showed that circFOXP1 may affect PKLR degradation in GBC.

Based on these findings, we hypothesized that circFOXP1 may enhance the capacity of PTBP1 to bind to PKLR mRNA and protect it from mRNA decay and 


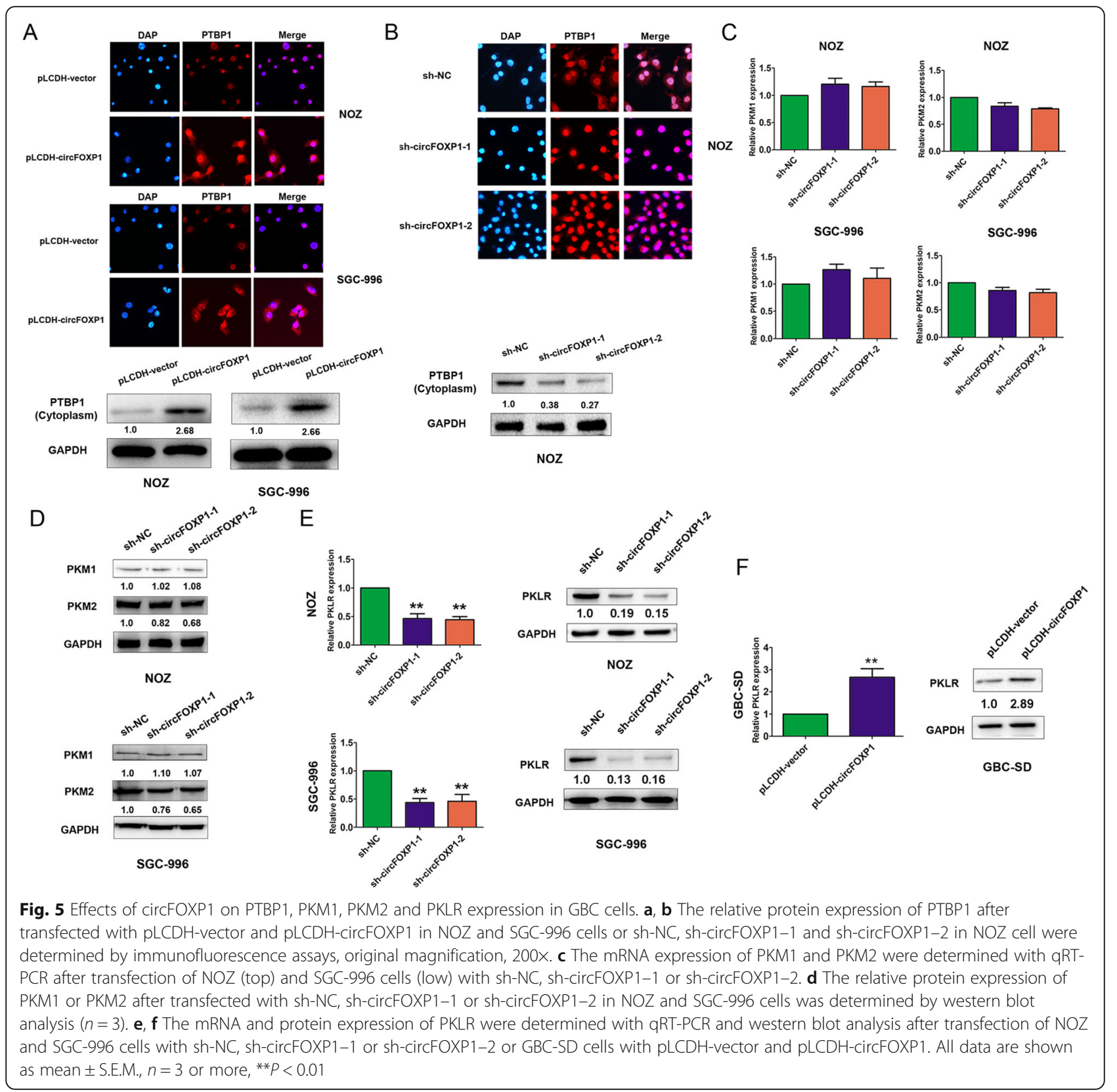

consequently increasing the PKLR protein levels. To test this hypothesis, we utilized a RIP assay to detect the association between PKLR mRNA and PTBP1. The results demonstrated that PTBP1 could bind to PKLR mRNA in NOZ and SGC-996 cells (Fig. 6b). Using the online prediction software RBP map (http://rbpmap.technion.ac.il/ ), we found that PTBP1 could bind to the 3'UTR region and coding region (CDS) of PKLR mRNA (UCUU binding bites) (Additional file 9: Figure S6A-6B). Furthermore, an RNA pull-down assay with the 3'UTR region or CDS region of PKLR biotin-labeled RNA probe confirmed that PTBP1 directly bound to PKLR mRNA in NOZ and SGC-996 cells (Fig. 6c). A RIP assay with
PTBP1 antibody demonstrated that downregulation of circFOXP1 reduced the enrichment of PKLR mRNA in NOZ and SGC-996 cells, but enhanced the enrichment of PKLR mRNA after overexpression of circFOXP1 in GBC-SD cells (Fig. 6d-e). We further detected the effects of PTBP1 knockdown on PKLR expression. Downregulation of PTBP1 reduced both PKLR mRNA and protein in NOZ and SGC-996 cells, and knockdown of PTBP1 abrogated the effect of overexpressed circFOXP1 on PKLR expression in GBC-SD cells (Additional file 10: Figure S7A and Fig. 6f). These results revealed that circFOXP1 promoted PKLR mRNA expression by interacting with PTBP1 in GBC cells. 


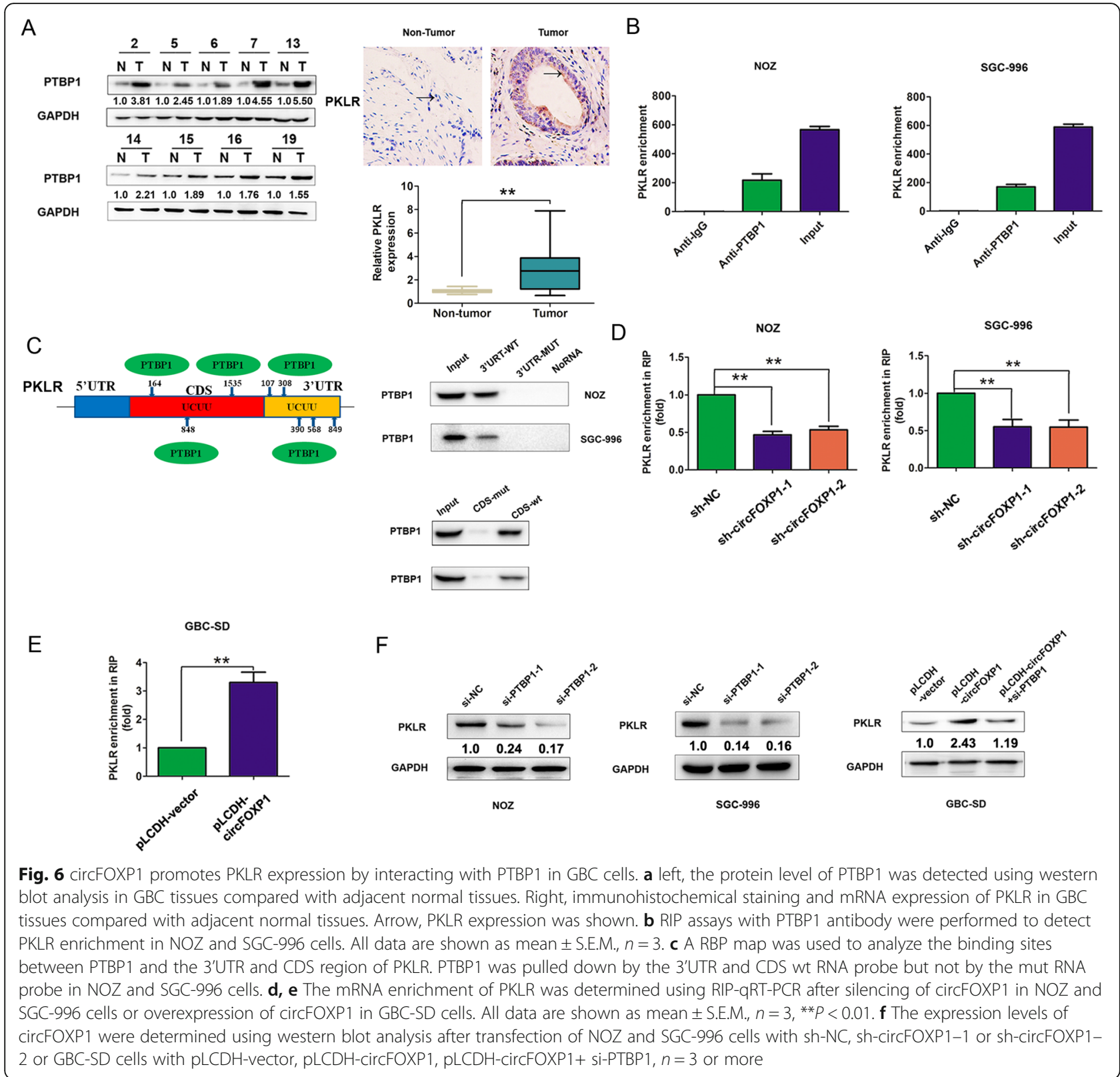

\section{The circFOXP1 binds to miR-370 targeting PKLR in GBC} cells

Endogenous circRNAs have been found to act as microRNA (miRNA) sponges in human cancers [38]. As circFOXP1 was predominantly localized in the cytoplasm, we hypothesized that circFOXP1 could also regulate PKLR expression by binding to specific miRNAs. To confirm this hypothesis, we performed a search for miRNAs that have complementary base pairing with circFOXP1 using the online software tools circinteractome (http://circinter actome.nia.nih.gov) and circRNAs from RNA sequencing targeted miRNAs predicted analysis by miRanda (www. microrna.org) (Fig. 7a, left, Additional file 11: Table S3). The results from RNA sequencing showed that miR-370 could form complementary base pairing with circFOXP1 and PKLR, respectively (Fig. 7a, right, and Fig. 7c). To verify that circFOXP1 could bind to miR-370, the wild-type (WT) and two mutant-type (MUT) circFOXP1 reporter vectors were constructed. We observed that a miR-370 mimic significantly reduced the luciferase activity of the circFOXP1-WT-1 (37\%) and circFOXP1-WT-2 (33\%) reporter vectors but not that of circFOXP1-MUT-1 or circFOXP1-MUT-2, which suggested that miR-370 was a target of circFOXP1 in a sequence-specific manner (Fig. 7b). To further clarify the regulatory association between circFOXP1 and the 3'UTR of PKLR, we constructed wild-type (WT) and mutant-type (MUT) PKLR 3'UTR reporter vectors (Fig. 7c). The results revealed 


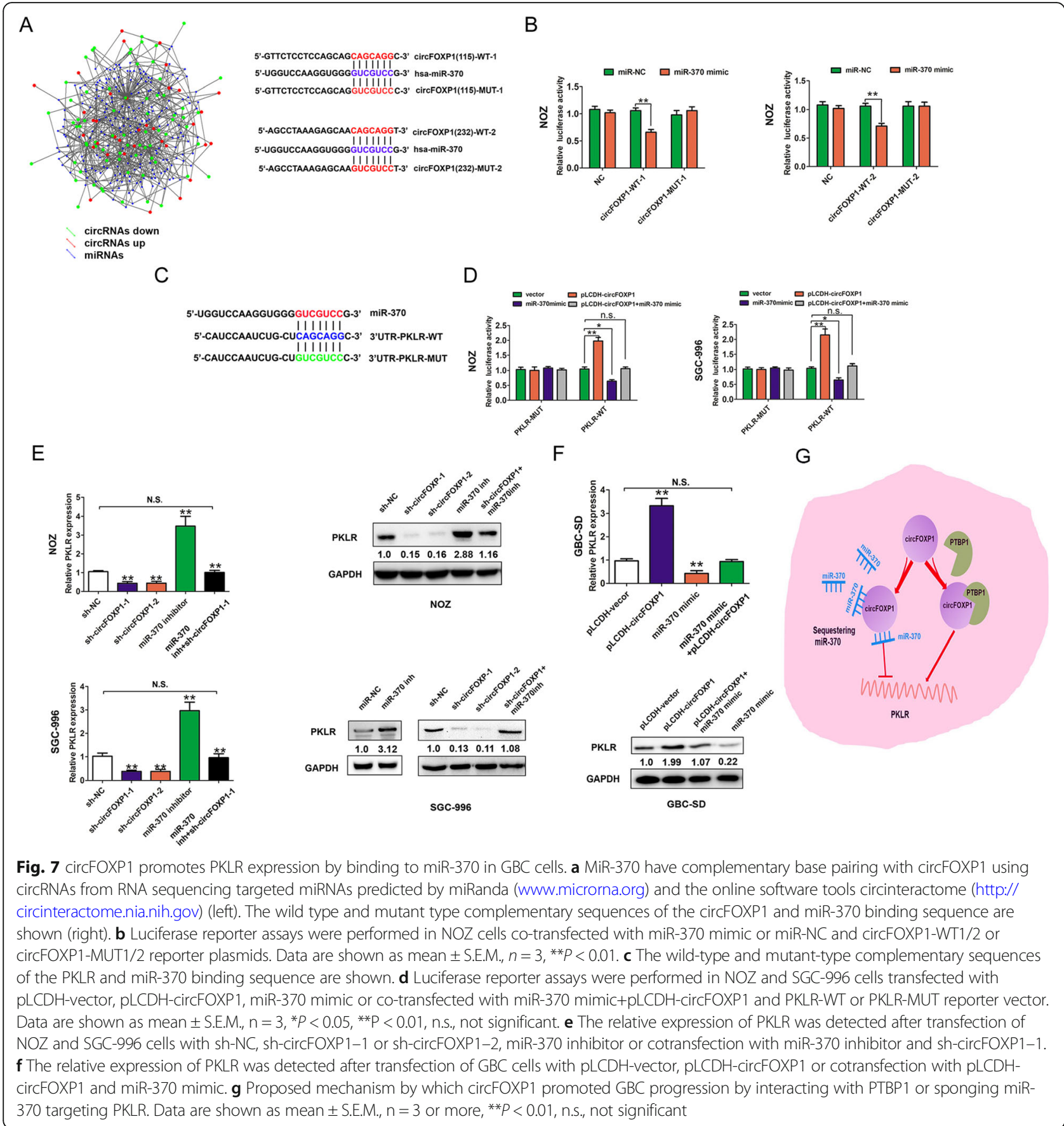

that the miR-370 mimic significantly reduced the luciferase activity of the WT PKLR 3'UTR reporter vector but not that of the mutant PKLR 3'UTR reporter. Cotransfection with the WT PKLR 3'UTR and miR-370 mimic and pLCDH-circFOXP1 antagonized the effects (Fig. 7d). Furthermore, we confirmed that miR-370 expression was significantly downregulated in GBC tissues and cells, compared with adjacent normal tissues and H69 cell, respectively (Additional file 10: Figure S7B-7C). Moreover, an inverse correlation was observed between circFOXP1 and miR-370 expression in GBC tissues $(r=-0.45, P<$ 0.05, Additional file 10: Figure S7D). The PKLR mRNA and protein expression was downregulated after transfection of NOZ and SGC-996 cells with sh-circFOXP1, but was rescued by co-transfection with miR-370 inhibitor and shcircFOXP1 (Fig. 7e). Conversely, the PKLR mRNA and protein expression was increased after transfection of GBC-SD cells with a pLCDH-circFOXP1, but was rescued by co- 
transfection with pLCDH-circFOXP1 and miR-370 mimic (Fig. 7f). These results showed that circFOXP1 promoted PKLR expression by sponging miR-370 in GBC cells.

\section{Discussion}

The annotations of circRNAs by transcriptome-wide sequencing in various fundamental cellular biological processes including tumor development and progression have been thrust into the spotlight [39]. Fusion circRNAs (f-circRNAs) produced from transcribed exons of distinct genes were upregulated by cancer-associated chromosomal translocations and subsequently contributed to cellular transformation and enhanced cell viability and resistance upon tumor therapy [40]. In clear cell renal cell carcinoma, AR suppressed circHIAT1 expression by regulating the expression of its host gene (HIAT1) at the transcriptional level, which resulted in deregulation of miR195-5p/29a-3p/29c-3p, and increased CDC42 to enhance cell migration and invasion [41]. Silencing of circZKSCAN1 promoted cell proliferation, migration and invasion in hepatocellular carcinoma [42]. The circRNA CCDC66 promotes colon cancer growth and metastasis [43]. In spite of some advanced findings, the expression and possible carcinogenic involvement of circRNAs in GBC remains unknown. In this study, we first determined that circFOXP1 was highly upregulated in GBC tissues and cells, and higher circFOXP1 expression was implicated as an independent prognostic marker for OS in patients. Furthermore, upregulation of circFOXP1 expression had pleiotropic effects, including promotion of cell proliferation, migration, invasion, and cell cycle progression and inhibition of cell apoptosis in GBC. These results suggested that circFOXP1 acts as an oncogene and may serve as a prognostic biological marker in GBC.

Mechanistic studies confirmed that circFOXP1 exerted its tumor-promoting roles by modulating the Warburg effect through upregulation of PKLR expression by interacting with PTBP1, protecting PKLR from mRNA decay. PTBP1, an RNA-binding protein, exerts various molecular functions, including RNA metabolism, for example, by repressively regulating AS [44], controlling mRNA stability [34, 35], and determining mRNA localization [36]. The PKM1/M2 isoforms are generated through AS of two mutually exclusive exons, and this AS is controlled by PTBP1 [29]. Herein, we first detected the effects of circFOXP1 on PKM1 and PKM2 expression, and found that decreased circFOXP1 does not significantly change PKM1 expression and only partially affected the PKM2 protein levels, which had been reported in previous studies [32, 45]. Interestingly, we uncovered a novel regulation target of circFOXP1, PKLR, which was activated by upregulation of circFOXP1. We speculated that PTBP1 could affect PKLR mRNA levels by its typical characteristic nucleocytoplasmic shuttling. We demonstrated that circFOXP1 facilitated
PTBP1 nuclear to cytoplasmic translocation, resulting in enhancement of the control of PKLR mRNA stability. PTBP1 is critical for post-transcriptional upregulation of proinsulin and other granule proteins biosynthesized shortly after glucose stimulation by stabilizing mRNAs encoding proteins of secretory granules [46, 47]. We found that PTBP1 bound to the 3'UTR and CDS region of PKLR mRNA. Silencing circFOXP1 decreased the ability of PTBP1 to bind PKLR mRNA, which significantly suppressed the Warburg effects in GBC. This is consistent with a previous study that reported PTBP1-mediated posttranscriptional upregulation of proinsulin [46]. Because PTBP1 is mainly located in the nucleus, but circFOXP1 is located in the cytoplasm, we speculated that circFOXP1 could serve as a protein scaffold to recruit not only PTBP1 but also other proteins to the circFOXP1-PTBP1 complex to affect PKLR mRNA expression. Future studies may be necessary to elucidate how circFOXP1 affects the ability of PTBP1 to bind target PKLR mRNA.

Several circRNAs have been found to function as miRNA "sponges" to counteract miRNA-mediated repression of mRNA. CiRS-7, which acts as a designated miR-7 inhibitor, harbors more than 70 conventional miR-7 binding sites and provides a conceptual mechanistic understanding of competing RNA (ceRNA) networks [11]. The circHIPK3 regulates cell growth by binding to miR-124 and inhibiting miR-124 activity [12]. circRNA HRCR functions as an endogenous miR-223 sponge to sequester and inhibit miR223 activity, which protects the heart from pathological hypertrophy and heart failure [48]. The circPVT1 promotes gastric cancer cell proliferation by acting as a sponge for members of the miR-125 family [49]. Based on the findings, we demonstrated that miR-370 was a direct target of circFOXP1. Upregulation of circFOXP1 promoted PKLR expression by sponging miR-370 in GBC cells.

\section{Conclusions}

Together, our study is the first to reveal that circFOXP1 was upregulated in GBC and promoted the tumor progression in GBC cells by interacting with PTBP1 or sponging miR-370 targeting PKLR (Fig. 7g). These findings have significant implications for our understanding of GBC pathogenesis and provide a target for GBC treatment.

\section{Supplementary information}

Supplementary information accompanies this paper at https://doi.org/10. 1186/s12943-019-1078-z.

Additional file 1. Supplementary materials and methods.

Additional file 2. The sequences of all primers and oligonucleotide used in the study.

Additional file 3. Relative expression levels of several circRNAs in GBC tissues are shown.

Additional file 4. CircFOXP1 is identified in GBC cells. 
Additional file 5. CircFOXP1 promotes cell migration and invasion in GBC.

Additional file 6. Proteins are identified by LC-MS that specifically associated with circFOXP1.

Additional file 7. Effects of PKLR on Warburg effect in GBC cells.

Additional file 8. CircFOXP1 affects PKLR expression in GBC cells.

Additional file 9. PTBP1 binds to $3^{\prime} U T R$ and CDS region of human PKLR mRNA.

Additional file 10. Expression of circFOXP1 is negatively associated with miR-370 in GBC tissues and cells.

Additional file 11. CircRNAs from RNA sequencing targeted miRNAs.

\section{Abbreviations}

AS: Alternative splicing; CDK2: cyclin-dependent kinase 2;

CDNA: complementary DNA; ceRNA: competing endogenous RNA; circFOXP1: circular RNA FOXP1; circRNAs: circular RNAs; FISH: Fluorescence in situ hybridization; GBC: Gallbladder cancer; LC-MS: Liquid ChromatographyMass Spectrometry; MUT: Mutant-type; ncRNA: non-coding RNA; OS: Overall survival; PCNA: Proliferating cell nuclear antigen; PKLR: pyruvate kinase, liver and RBC; qRT-PCR: quantitative real-time PCR; RIP: RNA immunoprecipitation; SDS/PAGE: SDS-polyacrylamide gel electrophoresis; shRNA: short hairpin RNA; WT: wild-type

\section{Acknowledgments}

We thank for the members of Department of General Surgery, Xinhua Hospital, Shanghai Jiao Tong University School of Medicine for helpful discussions.

\section{Authors' contributions}

SW and ZQ study concept and design, acquisition of data, analysis and interpretation of data, statistical analysis, project funding, and drafting of the manuscript. YZ, QC, MM, and LYJ acquisition of data, material support, and statistical analysis. SW, MW, DZ, ZT and JDW, study concept and design, critical revision of the manuscript for important intellectual content, analysis and interpretation of data, perform the experiments. All authors read and approved the final manuscript.

\section{Funding}

This work was supported by the National Natural Science Foundation of China (grant number 81572297 and 81802315) and Sponsored by Shanghai Sailing Program (grant number18YF1416000).

\section{Availability of data and materials}

The datasets used and analyzed during the current study are available from the corresponding author on reasonable request.

\section{Ethics approval and consent to participate}

The study procedure was approved by the Human Ethics Committee of Xinhua Hospital and All patients signed consent forms. The study protocol was approved by the Animal Care and Use committee of Xinhua Hospital (approval ID: 2014041).

\section{Consent for publication}

Not applicable.

\section{Competing interests}

The authors declare that they have no competing interests.

\section{Author details}

'Department of General Surgery, Xinhua Hospital, Shanghai Jiao Tong University School of Medicine, No.1665 Kong jiang Road, Yangpu District, Shanghai 200000, China. ${ }^{2}$ Department of Molecular Oncology \& Biliary Tract Surgery, Eastern Hepatobiliary Surgery Hospital, National Center of Liver Cancer, Second Military Medical University, Shanghai, China. ${ }^{3}$ Department of Gastric Cancer and Soft Tissue Sarcoma, Fudan University Shanghai Cancer Center, Shanghai, China.
Received: 2 July 2019 Accepted: 16 September 2019

Published online: 17 October 2019

\section{References}

1. Kakaei F, Beheshtirouy S, Nejatollahi SM, Zarrintan S, Mafi MR. Surgical treatment of gallbladder carcinoma: a critical review. Updat Surg. 2015; 67:339-51.

2. Lazcano-Ponce EC, Miquel JF, Munoz N, Herrero R, Ferrecio C, Wistuba II, Alonso de Ruiz P, Aristi Urista G, Nervi F. Epidemiology and molecular pathology of gallbladder cancer. CA Cancer J Clin. 2001;51:349-64.

3. Kanthan R, Senger $J$, Ahmed S, Kanthan SC. Gallbladder Cancer in the 21st century. J Oncol. 2015;2015:967472.

4. Caldow Pilgrim CH, Groeschl RT, Quebbeman EJ, Gamblin TC. Recent advances in systemic therapies and radiotherapy for gallbladder cancer. Surg Oncol. 2013;22:61-7.

5. Horgan AM, Amir E, Walter T, Knox JJ. Adjuvant therapy in the treatment of biliary tract cancer: a systematic review and meta-analysis. J Clin Oncol. 2012;30:1934-40.

6. Li Y, Zheng Q, Bao C, Li S, Guo W, Zhao J, Chen D, Gu J, He X, Huang S. Circular RNA is enriched and stable in exosomes: a promising biomarker for cancer diagnosis. Cell Res. 2015;25:981-4.

7. Salzman J. Circular RNA expression: its potential regulation and function. Trends Genet. 2016;32:309-16.

8. Lasda E, Parker R. Circular RNAs: diversity of form and function. Rna. 2014;20:1829-42.

9. Li J, Yang J, Zhou P, Le Y, Zhou C, Wang S, Xu D, Lin HK, Gong Z. Circular RNAs in cancer: novel insights into origins, properties, functions and implications. Am J Cancer Res. 2015;5:472-80.

10. Qu S, Yang X, Li X, Wang J, Gao Y, Shang R, Sun W, Dou K, Li H. Circular RNA: a new star of noncoding RNAs. Cancer Lett. 2015;365:141-8.

11. Hansen TB, Kjems J, Damgaard CK. Circular RNA and miR-7 in cancer. Cancer Res. 2013;73:5609-12.

12. Zheng Q, Bao C, Guo W, Li S, Chen J, Chen B, Luo Y, Lyu D, Li Y, Shi $\mathrm{G}$, et al. Circular RNA profiling reveals an abundant circHIPK3 that regulates cell growth by sponging multiple miRNAs. Nat Commun. 2016;7:11215.

13. Du WW, Yang W, Liu E, Yang Z, Dhaliwal P, Yang BB. Foxo3 circular RNA retards cell cycle progression via forming ternary complexes with p21 and CDK2. Nucleic Acids Res. 2016;44:2846-58.

14. Mathupala SP, Ko YH, Pedersen PL. Hexokinase-2 bound to mitochondria: cancer's stygian link to the "Warburg effect" and a pivotal target for effective therapy. Semin Cancer Biol. 2009;19:17-24.

15. Nie H, Li J, Yang XM, Cao QZ, Feng MX, Xue F, Wei L, Qin W, Gu J, Xia Q, Zhang ZG. Mineralocorticoid receptor suppresses cancer progression and the Warburg effect by modulating the miR-338-3p-PKLR axis in hepatocellular carcinoma. Hepatology. 2015;62:1145-59.

16. Yang Y, Zhu G, Dong B, Piao J, Chen L, Lin Z. The NQ01/PKLR axis promotes lymph node metastasis and breast cancer progression by modulating glycolytic reprogramming. Cancer Lett. 2019;453:170-83.

17. Rybak-Wolf A, Stottmeister C, Glazar P, Jens M, Pino N, Giusti S, Hanan M, Behm M, Bartok O, Ashwal-Fluss R, et al. Circular RNAs in the mammalian brain are highly abundant, conserved, and dynamically expressed. Mol Cell. 2015;58:870-85.

18. Glazar P, Papavasileiou P, Rajewsky N. circBase: a database for circular RNAs. Rna. 2014;20:1666-70.

19. Wang SH, Zhang WJ, Wu XC, Weng MZ, Zhang MD, Cai Q, Zhou D, Wang JD, Quan ZW. The IncRNA MALAT1 functions as a competing endogenous RNA to regulate MCL-1 expression by sponging miR-363-3p in gallbladder cancer. J Cell Mol Med. 2016;20:2299-308.

20. Wang P, Xue Y, Han Y, Lin L, Wu C, Xu S, Jiang Z, Xu J, Liu Q, Cao X. The STAT3-binding long noncoding RNA Inc-DC controls human dendritic cell differentiation. Science. 2014;344:310-3.

21. Wang SH, Ma F, Tang ZH, Wu XC, Cai Q, Zhang MD, Weng MZ, Zhou D, Wang JD, Quan ZW. Long non-coding RNA H19 regulates FOXM1 expression by competitively binding endogenous miR-342-3p in gallbladder cancer. J Exp Clin Cancer Res. 2016;35:160.

22. Ferreiro-Neira I, Torres NE, Liesenfeld LF, Chan CH, Penson T, Landesman Y, Senapedis W, Shacham S, Hong TS, Cusack JC. XPO1 inhibition enhances radiation response in preclinical models of rectal Cancer. Clin Cancer Res. 2016;22:1663-73. 
23. Flori M, Schmid CA, Sumrall ET, Tzankov A, Law CW, Robinson MD, Muller A. The hematopoietic oncoprotein FOXP1 promotes tumor cell survival in diffuse large B-cell lymphoma by repressing S1PR2 signaling. Blood. 2016;127:1438-48.

24. Mebratu Y, Tesfaigzi Y. How ERK1/2 activation controls cell proliferation and cell death: is subcellular localization the answer? Cell Cycle. 2009;8:1168-75.

25. Cui CP, Wong CC, Kai AK, Ho DW, Lau EY, Tsui YM, Chan LK, Cheung TT, Chok KS, Chan AC, et al. SENP1 promotes hypoxia-induced cancer stemness by HIF-1alpha deSUMOylation and SENP1/HIF-1alpha positive feedback loop. Gut. 2017;66:2149-59.

26. Ying Z, Tian H, Li Y, Lian R, Li W, Wu S, Zhang HZ, Wu J, Liu L, Song J, et al. CCT6A suppresses SMAD2 and promotes prometastatic TGF-beta signaling. J Clin Invest. 2017;127:1725-40.

27. Suzuki H, Tsukahara T. A view of pre-mRNA splicing from RNase $R$ resistant RNAs. Int J Mol Sci. 2014;15:9331-42.

28. Warburg O. On the origin of cancer cells. Science. 1956;123:309-14.

29. Chen M, Zhang J, Manley JL. Turning on a fuel switch of cancer: hnRNP proteins regulate alternative splicing of pyruvate kinase mRNA. Cancer Res. 2010;70:8977-80.

30. Christofk HR, Vander Heiden MG, Wu N, Asara JM, Cantley LC. Pyruvate kinase $M 2$ is a phosphotyrosine-binding protein. Nature. 2008;452:181-6.

31. Lu W, Cao Y, Zhang Y, Li S, Gao J, Wang XA, Mu J, Hu YP, Jiang L, Dong P, et al. Up-regulation of PKM2 promote malignancy and related to adverse prognostic risk factor in human gallbladder cancer. Sci Rep. 2016;6:26351.

32. Calabretta S, Bielli P, Passacantilli I, Pilozzi E, Fendrich V, Capurso G, Fave GD, Sette C. Modulation of PKM alternative splicing by PTBP1 promotes gemcitabine resistance in pancreatic cancer cells. Oncogene. 2016;35:2031-9.

33. Nguyen A, Loo JM, Mital R, Weinberg EM, Man FY, Zeng Z, Paty PB, Saltz L, Janjigian $Y Y$, de Stanchina E, Tavazoie SF. PKLR promotes colorectal cancer liver colonization through induction of glutathione synthesis. J Clin Invest. 2016:126:681-94.

34. Tillmar L, Carlsson C, Welsh N. Control of insulin mRNA stability in rat pancreatic islets. Regulatory role of a 3'-untranslated region pyrimidine-rich sequence. J Biol Chem. 2002;277:1099-106.

35. Cui J, Placzek WJ. PTBP1 modulation of MCL1 expression regulates cellular apoptosis induced by antitubulin chemotherapeutics. Cell Death Differ. 2016;23:1681-90.

36. Cote CA, Gautreau D, Denegre JM, Kress TL, Terry NA, Mowry KL. A Xenopus protein related to hnRNP I has a role in cytoplasmic RNA localization. Mol Cell. 1999;4:431-7.

37. Ge Z, Quek BL: Polypyrimidine tract binding protein 1 protects mRNAs from recognition by the nonsense-mediated mRNA decay pathway. 2016, 5.

38. Cortes-Lopez M, Miura P. Emerging functions of circular RNAs. Yale J Biol Med. 2016;89:527-37.

39. Barrett SP, Salzman J. Circular RNAs: analysis, expression and potential functions. Development. 2016;143:1838-47.

40. Guarnerio J, Bezzi M, Jeong JC, Paffenholz SV, Berry K, Naldini MM, Lo-Coco F, Tay $Y$, Beck AH, Pandolfi PP. Oncogenic role of fusion-circRNAs derived from Cancer-associated chromosomal translocations. Cell. 2016:165:289-302.

41. Wang K, Sun Y, Tao W, Fei X, Chang C. Androgen receptor (AR) promotes clear cell renal cell carcinoma ( $C C R C C$ ) migration and invasion via altering the circHIAT1/miR-195-5p/29a-3p/29c-3p/CDC42 signals. Cancer Lett. 2017;394:1.

42. Yao Z, Luo J, Hu K, Lin J, Huang H, Wang Q, Zhang P, Xiong Z, Huang Z, He C, et al. ZKSCAN1 gene and its related circular RNA (circZKSCAN1) both inhibit hepatocellular carcinoma cell growth, migration and invasion but through different signaling pathways. Mol Oncol. 2017;11:422-37.

43. Hsiao KY, Lin YC, Gupta SK, Chang N, Yen L, Sun HS, Tsai SJ. Non-coding effects of circular RNA CCDC66 promote colon cancer growth and metastasis. Cancer Res. 2017;77:2339-50.

44. Cheung HC, Hai T, Zhu W, Baggerly KA, Tsavachidis S, Krahe R, Cote GJ. Splicing factors PTBP1 and PTBP2 promote proliferation and migration of glioma cell lines. Brain. 2009;132:2277-88.

45. Dayton TL, Jacks T, Vander Heiden MG: PKM2, cancer metabolism, and the road ahead. 2016, 17:1721-1730.

46. Knoch KP, Bergert $H$, Borgonovo B, Saeger HD, Altkruger A, Verkade $P$, Solimena M. Polypyrimidine tract-binding protein promotes insulin secretory granule biogenesis. Nat Cell Biol. 2004;6:207-14.

47. Knoch KP, Nath-Sain S, Petzold A, Schneider H, Beck M, Wegbrod C, Sonmez A, Munster C, Friedrich A, Roivainen M, Solimena M. PTBP1 is required for glucose-stimulated cap-independent translation of insulin granule proteins and Coxsackieviruses in beta cells. Mol Metab. 2014:3:518-30.
48. Wang K, Long B, Liu F, Wang JX, Liu CY, Zhao B, Zhou LY, Sun T, Wang M, Yu T, et al. A circular RNA protects the heart from pathological hypertrophy and heart failure by targeting miR-223. Eur Heart J. 2016;37:2602-11.

49. Chen J, Li Y, Zheng Q, Bao C, He J, Chen B, Lyu D, Zheng B, Xu Y, Long Z, et al. Circular RNA profile identifies circPVT1 as a proliferative factor and prognostic marker in gastric cancer. Cancer Lett. 2017;388:208-19.

\section{Publisher's Note}

Springer Nature remains neutral with regard to jurisdictional claims in published maps and institutional affiliations.
Ready to submit your research? Choose BMC and benefit from:

- fast, convenient online submission

- thorough peer review by experienced researchers in your field

- rapid publication on acceptance

- support for research data, including large and complex data types

- gold Open Access which fosters wider collaboration and increased citations

- maximum visibility for your research: over $100 \mathrm{M}$ website views per year

At BMC, research is always in progress.

Learn more biomedcentral.com/submissions 